\title{
Taxonomic Study on the Subfamily Curculioninae (Col., Curculionidae) in East Azarbaijan Province with one Species as New Record for Iran
}

\author{
R. RAHATI ${ }^{1}$, J. NOZARI $^{1}$ and S. KHAGHANINIA ${ }^{2 *}$ \\ ${ }^{1}$ Department of Plant Protection, College of Agriculture, University of Tehran, Karaj, Iran \\ ${ }^{2}$ Department of Plant Protection, Faculty of Agriculture, University of Tabriz, 51664, Tabriz, Iran
}

(Received: 22 April 2017; accepted: 12 May 2017)

\begin{abstract}
The present faunistic study was performed in East Azarbaijan province, northwest of Iran, in 20152016. A total of eleven species belonging to four genera of the subfamily Curculioninae were studied. Rhinusa eversmanni Rosenschoeld, 1838 is recorded for the first time from Iran. Photos of adult habitus of the newly recorded species along with a key to the Iranian species of the snout beetles identified in this study are provided.
\end{abstract}

Keywords: Curculionidae, Curculioninae, Rhinusa, new record, East Azarbaijan, Iran.

The Curculionidae is the largest family in the animal kingdom, including about 48,000 described species worldwide. Their size is small to very large (1-60 mm), they come with various habitats, shapes and colors. Both adults and larvae feed on plants and the majority of the species are endophytic, i.e. live in the living tissues of the host plants (Anderson, 2002; Marvaldi and Lanteri, 2005; Oberprieler et al., 2007).

The subfamily Curculioninae is one of the largest subfamilies of Curculionidae including approximately 350 genera and 4,500 species. Tychiini and Mecinini are among the largest tribes of the subfamily represented by 600 and 260 valid species, respectively. Distribution of both tribes primarily includes the whole Palaearctic and Afrotropical regions, whereas Tychiini is widely distributed also in America (Caldara et al., 2014). Concerning Palaearctic Tychiini, members of Tychius Germar, 1817 usually live on the Fabaceae family (Hoffmann, 1954; Caldara, 1990). The majority of species of Tychius feed on seeds. Almost all species of Sibinia feed on seeds of Caryophyllaceae, Plumbaginaceae (including Staticoideae), Paronychiaceae and Thymeleaceae (Hoffmann, 1954; Caldara, 1985). Concerning Palaearctic Mecinini, species of Rhinusa Stephens, 1829 usually live on species of Scrophulariaceae, with two groups occurring on two closely related plant genera, Scrophularia and Verbascum (Caldara, 2014). The majority of the Mecinus species Germar, 1821, live on tribes Plantagineae and Antirrhineae of the family Plantaginaceae (Caldara and Fogato, 2013).

* Corresponding author; e-mail: skhaghaninia@gmail.com 
Contributions to the fauna of Curculioninae in Iran were given by Borumand (1998), Modarres Awal (2010), Legalov et al. (2010) and Abad et al. (2015). Borumand (1998) reported 287 species of the superfamily Curculionoidea, among which 15 species belong to the subfamily Curculioninae. Modarres Awal (2010) in his book reported 189 species of the superfamily Curculionoidea, among which 10, 2, 0, species belong to the genera Tychius, Mecinus, Rhinusa of Curculioninae, respectively. Legalov et al. (2010) reported 61 new record of superfamily Curculionoidea, including one species of Tychius and one species of Rhinusa. Finally Abad et al. (2015) described 29 species belonging to the genera Mecinus Germar, 1821, Gymnetron Schoenherr, 1825, Rhinusa Stephens, 1829, Cleopomiarus Pierce, 1919, Tychius Germar, 1817 and Sibinia Germar, 1817 with ecological notes on them. Of them 11 species represented new records for Iranian fauna.

As the fauna of this subfamily in Iran has been poorly studied, the aim of the present work is a preliminary discussion on the zoogeography and fauna of the subfamily Curculioninae in the mentioned area.

\section{Materials and Methods}

All specimens were collected by sweep-netting in East Azarbaijan province during 2015-2016. In order to prepare the male genitalia, the posterior part of the abdomen was removed and boiled in $10 \%$ solution of $\mathrm{KOH}$ (potassium hydroxide) for 45-60 seconds, then transferred into acetic acid for 10-20 seconds and later washed in distilled water. The genitalia were stored in $0.5 \mathrm{ml}$ microvials containing glycerin. Classification and nomenclature of curculionids were followed as suggested by Roberto Caldara. All specimens are kept in the Jalal Afshar Zoological Museum, University of Tehran, Karaj, Iran.

\section{Results}

In total eleven species belonging to four genera of the subfamily Curculioninae were identified, of which Rhinusa eversmanni Rosenschoeld, 1838 is newly recorded from Iran. Tychius breviusculus Desbrochers des Loges, 1873 and Tychius urbanus Faust, 1885 are newly recorded form the province.

\section{Key to the studied species}

$$
\text { adapted from Hoffman (1954) }
$$

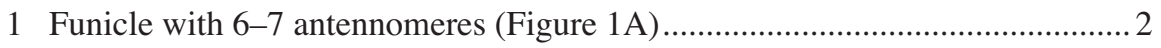

- Funicle with 4-5 antennomeres (Figure 1B) ..................................................... 3

2 Funicle with 6-7 antennomeres, pygidium covered by elytra (Figure 2). Tychius..... 


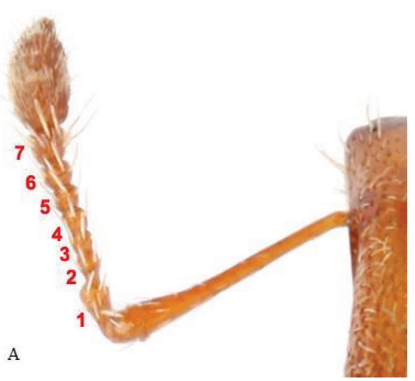

B

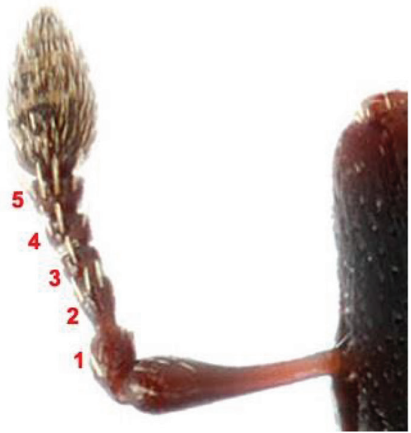

Fig. 1. Antennal funicle, A) Tychiini (with 7 antennomeres)

B) Mecinini (with 5 antennomeres) (Photo by KV Makarov)

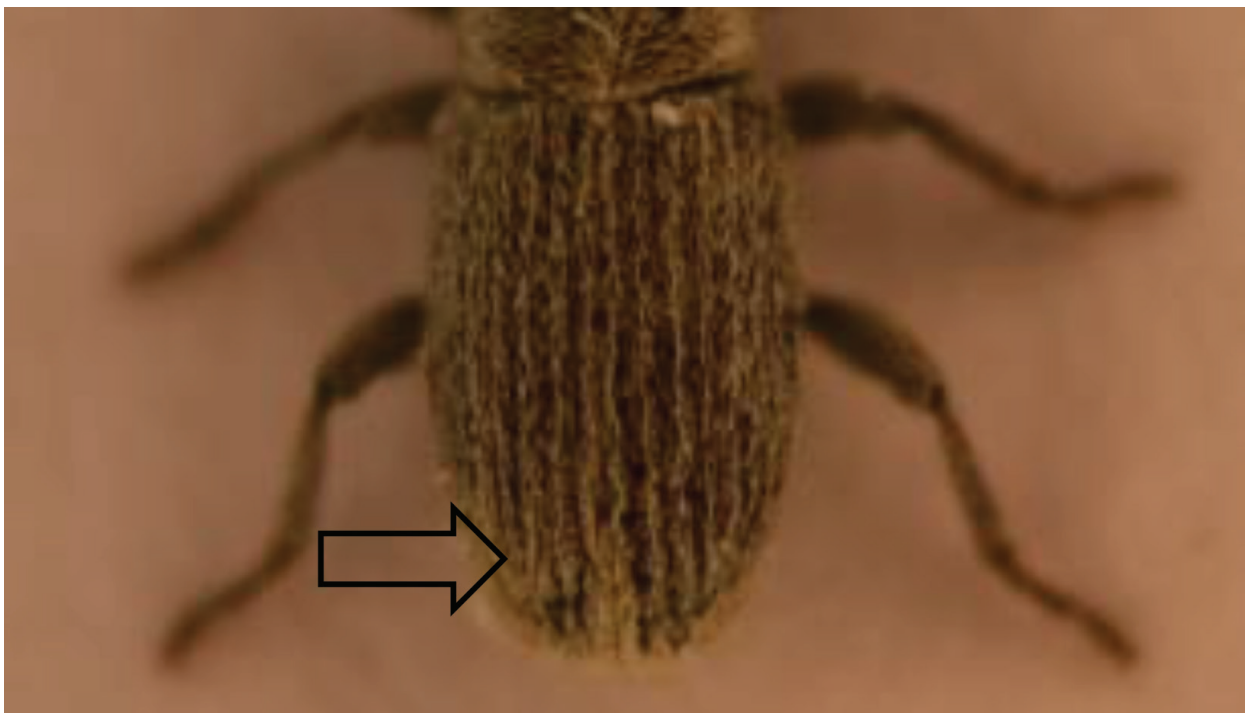

Fig. 2. Pygidium covered by elytra

- Funicle with 6 antennomeres, pygidium not covered by elytra (especially in male) (Figure 3) Sibinia

3 Elytral margin at apex transverse to moderately directed outwards and covering large portion of pygidium; apical portion of ventral face of protibia directed outwards at apex; median lobe usually short (length/width $<3$ ) and with flagellum curled proximally. Mecinus 10

- Elytral margin at apex moderately directed inwards (Figure 4) and leaving pygidium mostly uncovered; apical portion of ventral face of protibia not directed outwards at apex; median lobe usually long (length/width $>3$ ) and with flagellum straight to S-shaped proximally 4

4 Elytral striae 3 and 8 joined at apex Gymnetron 


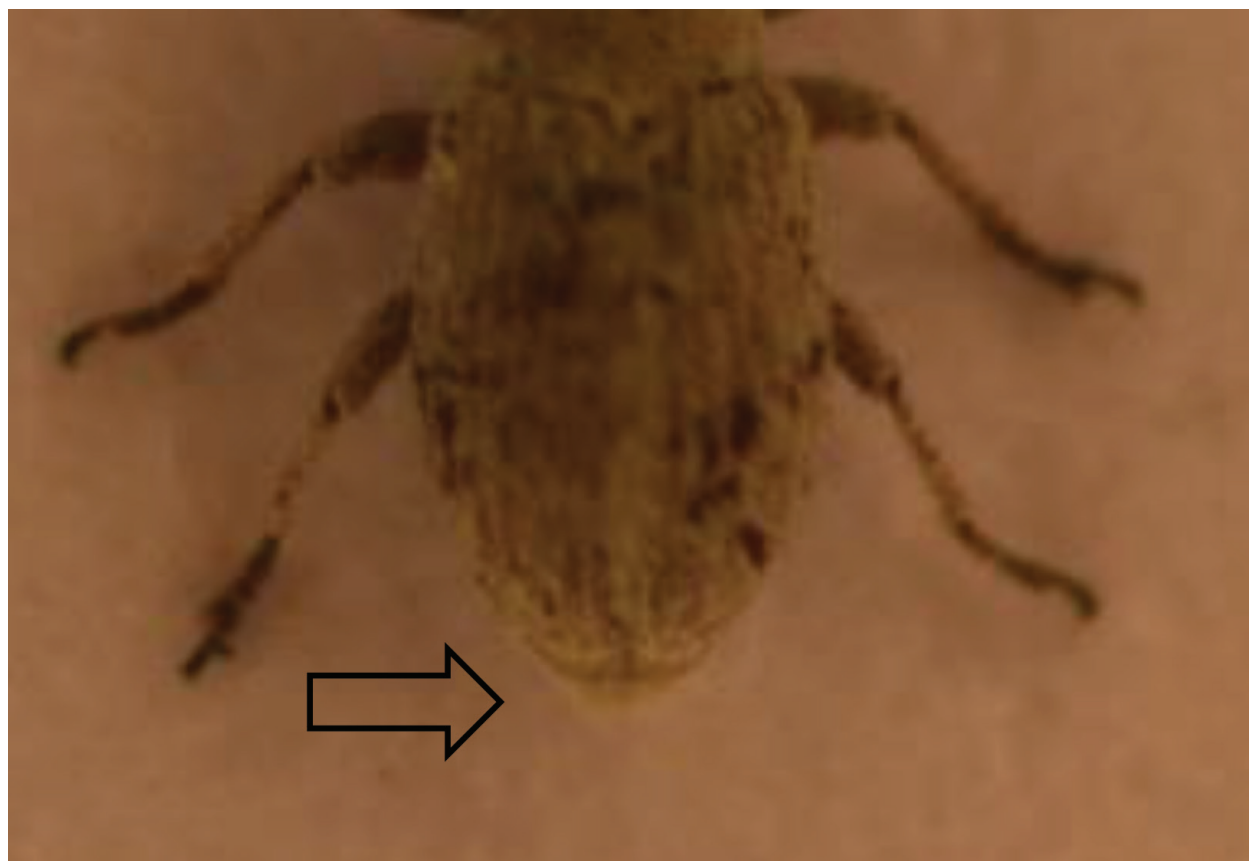

Fig. 3. Pygidium not covered by elytra

- Elytral striae 3 and 6 joined at apex (Figure 5)

Rhinusa. 11

5 Funicle with 6 antennomeres. Body length 1.5-2.3 mm T. picirostris

- Funicle with 7 antennomeres

6 Elytral intervals covered with variously coloured scales, Mesofemora and metafemora with distinct tooth. Body length 2.5-4.0 mm (Figure 6) T. quinquepunctatus

- Elytral intervals covered with unicolorous scales. Body length 1.3-2.7 mm ... 7

7 - Antennae reddish or reddish brown .......................................................... 8

- Some antennomeres of funicle and club darker ............................................. 9

8 Dorsal surface covered with, sparse, strongly elongated scales (6-8 times longer than wide).

Rostrum weakly tapered to antennal insertion. Body length $1.8-2.5 \mathrm{~mm}$.

T. junceus

- Upper surface covered with dense, slightly elongated scales (3-4 times longer than wide). Rostrum tapered to apex in lateral and dorsal views. Body length $2.3-2.7 \mathrm{~mm}$ T. aureolus

9 Elytra parallel-sided, hardly wider than pronotum. Dorsal scales not round. Body length 2-2.5 mm T. breviusculus

- Elytra not parallel-sited, wider than pronotum. Dorsal scales round. Body length 2-2.6 mm T. urbanus 
10 - Elytral integument reddish with at least two black oblique bands in basal half. Rostrum of female in lateral view straight in apical two-thirds. Body slightly narrower; elytra slightly longer. Rostrum more curved in basal third. Body length $1.7-2.1 \mathrm{~mm}$ M. labilis (Herbst)

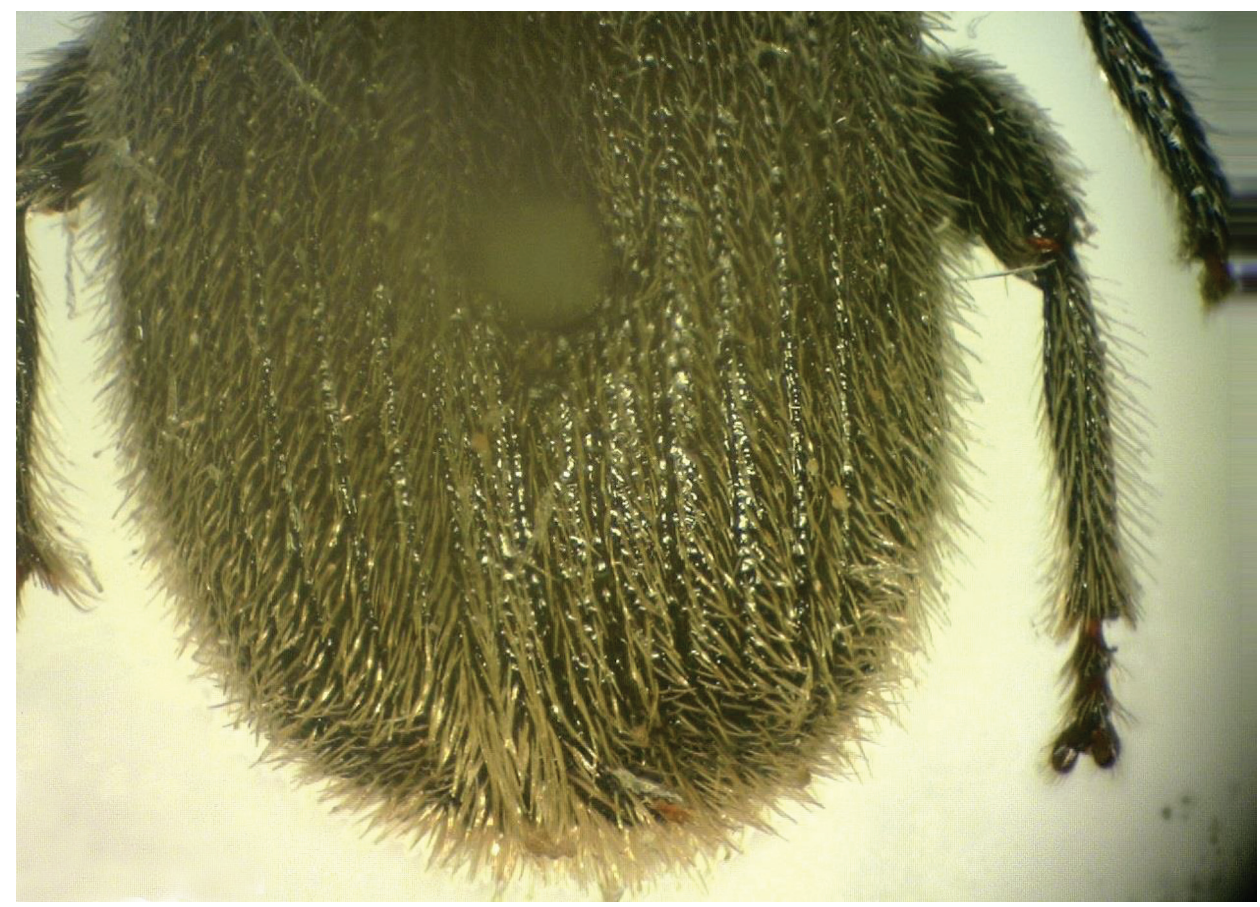

Fig. 4. Elytral margin at apex moderately directed inward

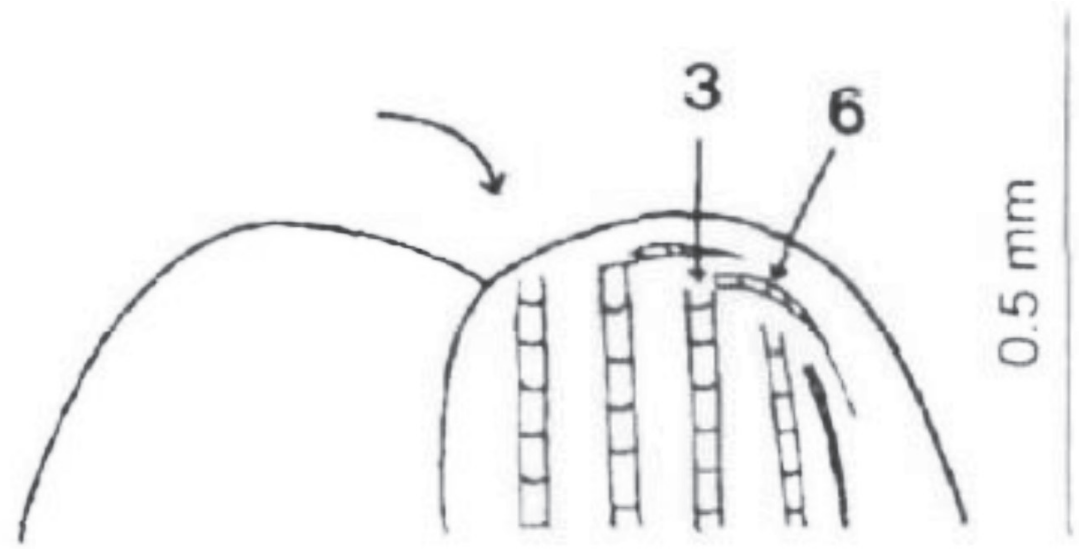

Fig. 5. Elytral striae 3 and 6 joined at apex (Caldara, 2001) 
- Elytral integument completely reddish to reddish with black parts never forming oblique bands in basal half. Rostrum of female in lateral view more or less curved from base to apex. Body length 1.3-1.8 mm M. pascuorum

11 - Rostrum slightly curved, long, in male almost as long as head and pronotum combined, in female much longer (Figure 7). Pronotum slightly convex ...R. eversmanni

- Rostrum almost straight, usually from antennal insertion to top clearly narrowed, rostrum length variable. Pronotum strongly transverse, with rounded sides, more narrowing anteriorly than posteriorly R. tetra
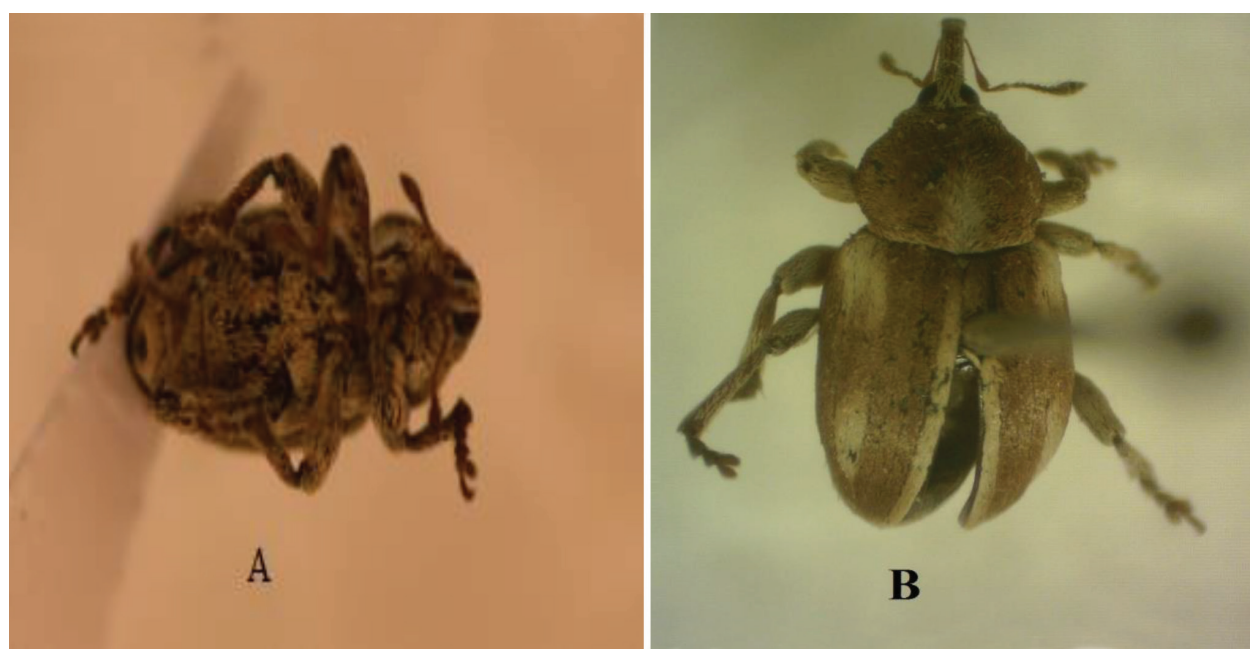

Fig. 6. Tychius quinquepunctatus, A) ventral view, B) dorsal view

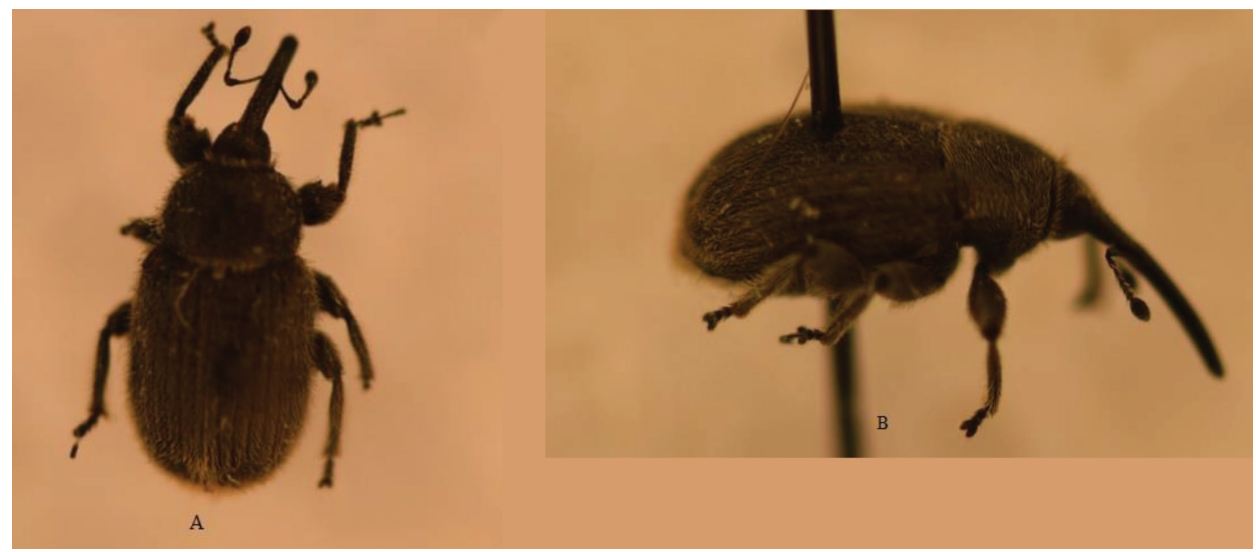

Fig. 7. Rhinusa eversmanni A) dorsal view, B) lateral view 


\section{The subfamily Curculioninae}

\section{Tribe Mecinini}

Genus: Mecinus Germar, 1821

Mecinus labilis (Herbst, 1975)

Material examined: (2 $\left.\sigma^{\top}, 5 q\right)$; Marand region (Alinjagh kandi village), $38^{\circ} 25^{\prime} 59.9^{\prime \prime} \mathrm{N} 46^{\circ} 04^{\prime} 57.8^{\prime \prime} \mathrm{E}$; 19. VII. 2015, leg. R. Rahati.

Distribution: Europe, Caucasus, Asia Minor.

Host plants: Plantago sp. (Isaev, 1994).

Mecinus pascuorum (Gyllenhal, 1813)

Material examined: (1 $\left.\sigma^{\top}, 3 q\right)$; Marand region (Alinjagh kandi village), 38²5'59.9"N 4604'57.8"E; 19. VII. 2015, leg. R. Rahati. On Plantago lanceolata L. (Plantaginaceae).

Distribution: Europe, Afrotropical Region, Australian Region, Nearctic Region, North Africa (Canary Islands), Asia (Russia: East and West Siberia, Iran, Israel, Turkey).

Host plants: Plantago sp. (Isaev, 1994).

Genus: Rhinusa Stephens, 1829

Rhinusa eversmanni (Rosenschöld, 1838)

Material examined: (1 $\sigma^{7}, 6$ O ); Osku region (Kandovan village), 3746'47.4"N 46²15'58.2"E; 13. IX. 2015, leg. R. Rahati. On Verbascum sp.

Distribution: part of Europe, Asia (Russia: East and West Siberia, Kazakhstan, Mongolia, Tajikistan, Turkey, Uzbekistan). New to Iran.

Host plants: Verbascum sp.

Diagnostic characters: Body length $2.7-3.4 \mathrm{~mm}$. Rostrum slightly curved, long, in male almost as long as head and pronotum combined, in female much longer. Structure of the rostrum similar to that of $R$. neta, but male antennae inserted slightly before middle, female antennae connected at a distance equal to 3.5 times the length. Antennomeres elongate, cylindrical, but their shape and size variable. Pronotum slightly convex. Elytra slightly longer than wide, clearly flattened above.

Rhinusa tetra (Fabricius, 1792)

Material examined: (40, 60$)$; Kaleybar region (Abbas Abad village), 38 53'53.5"N 46²8'44.8"E; 05. VII. 2015, leg. R. Rahati. On Verbascum speciosum.

Distribution: Europe, Nearctic Region, North Africa (Canary Islands, Algeria, Egypt), Asia (Afghanistan, Iran, Iraq, Israel, Kyrgyzstan, Kazakhstan, Lebanon, Nepal, Pakistan, Syria, Tajikistan, Turkmenistan, Turkey, India: Uttarakhand, Uttar Pradesh and Uzbekistan).

Host plants: Verbascum sp. (Isaev, 1994; Gholami Ghavam Abad et al., 2015); Verbascum speciosum. 


\section{Tribe Tychinii}

\section{Genus Sibinia Germar, 1817}

Sibinia reitteri Desbrochers des Loges, 1895

Material examined: ( $5 \sigma^{\top}, 10$ O ); Jolfa region; $38^{\circ} 50^{\prime} 41.0^{\prime \prime} \mathrm{N} 45^{\circ} 45^{\prime} 08.6 " \mathrm{E}, 08$. V. 2015, leg. R. Rahati.

Distribution: Armenia, Georgia, Iran, Iraq, Turkey.

Host plants: Unknown.

\section{Genus: Tychius Germar, 1817}

Tychius aurarius Boheman, 1843

Material examined: (207, 3 O ); Ajab Shir region (Yanchi village), 37³5'54.4"N 46¹1'01.1"E; 07. VII.2015; (2O) Chichekli (located in west part of Arasbaran forests),

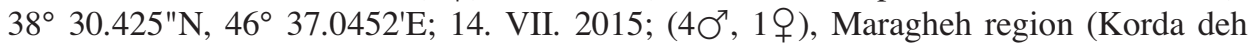
village), 3743'00.2"N 46²9'27.3"E; 16. VII. 2015, leg. R. Rahati.

Distribution: Europe, Azerbaijan, Albania, Armenia, Israel, Lebanon, Syria, Turkmenistan, Turkey, Iran.

Host plants: Unknown.

Tychius breviusculus Desbrochers des Loges, 1873

Material examined: (7 $\bigcirc^{\top}, 7$ ) $)$; Marand region (Eish Abad village), 38²2'34.0"N 45³7'47.1"E; 09. VII. 2015, leg. R. Rahati. On Alhagi maurorum Medik. (Fabaceae).

Distribution: Europe, Turkey, North Africa (Morocco), Siberia, Central Asia (Afghanistan, Northwest China, Iran), East Asia (North Korea, Mongolia, Russian Far East).

Host plants Melilotus sp. (Dieckmann, 1988; Egorov et al., 1996). Medicago sativa; Melilotus officinalis; Vicia sativa [Gholami Ghavam Abad et al., 2015]; Alhagi maurorum.

Tychius junceus (Reich, 1797)

Material examined: ( $3 \sigma^{\top}, 8$ ㅇ); Marand region (Eish Abad village), 38²2'34.0"N 45³7'47.1"E; 09. VII. 2015, leg. R. Rahati. On Alhagi maurorum.

Distribution: Europe, North Africa (Morocco (incl. Western Sahara)), Asia (Iran, Turkey, and Russia: west Siberia)

Host plants Lotus sp., Anthyllis sp., Trifolium sp., Melilotus sp., Medicago sp. (Dieckmann, 1988); Alhagi maurorum.

Tychius picirostris (Fabricius, 1787)

Material examined: (20', 1 O ); Marand region (Eish Abad village), 38²2'34.0"N 45³7'47.1"E; 09. VII. 2015, leg. R. Rahati.

Distribution: Europe, Siberia, Asia (Afghanistan, Cyprus, Russia: East and West Siberia, Far East, Iran, Japan, Kyrgyzstan, Kazakhstan, Mongolia, Tajikistan, Turkmenistan, Turkey, Uzbekistan).

Host plants Trifolium pratense, Trifolium resupinatum, Trifolium montanum, Trifolium repens (Abad et al., 2015). 
Tychius quinquepunctatus quinquepunctatus (Linnaeus, 1758)

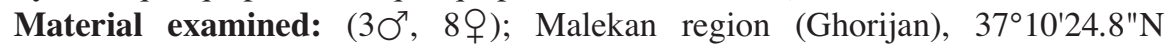
4607'47.5"E; 10. IX. 2015, leg. R. Rahati.

Distribution: Europe, North Africa (Algeria), Asia (Iran, Israel, Japan, Jordan, Kazakhstan, Syria, Turkey, and Russia: west Siberia).

Host plants Vicia sp., Lathyrus sp., Pisum sp. (Dieckmann, 1988; Egorov et al., 1996); Sainfoin sp. (Modarres Awal, 2010).

\section{Tychius urbanus Faust, 1885}

Material examined: (40', 6O)); Marand region (Eish Abad village), 38²2'34.0"N 45³7'47.1"E; 09. VII. 2015; on Alhagi maurorum Medik. (Fabaceae), (10', 3 O); Hash-

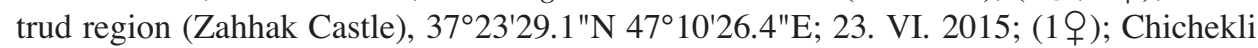
(located in west part of Arasbaran forests), 38 $30.425^{\prime \prime} \mathrm{N}, 46^{\circ} 37.0452^{\prime} \mathrm{E}$; 14. VII. 2015, leg. R. Rahati.

Distribution: North Africa (Egypt), Asia (Afghanistan, Iran, Jordan, Kazakhstan, Tajikistan, Turkmenistan, Turkey, Uzbekistan).

Host plants: Alhagi maurorum, Alhagi camelorum (Abad et al., 2015); Alhagi officinalis, new host.

\section{Discussion}

All of the studied species were collected from an East Azarbaijan province that has a rich fauna of Curculionidae. Curculionines tend to be associated with many herbaceous as well as some woody plants. Most have larvae that develop in reproductive structures such as fruits, seeds or flower buds; some also mine stems (Anderson, 2002). The host plant of Rhinusa eversmanni was previously thought to be Linaria vulgaris (Smreczyński, 1976), although it was supposed that it belongs to Scrophulariaceae. Our data confirm this hypothesis, since this weevil was collected on Verbascum sp. (Scrophulariaceae). Rhinusa tetra was collected on various species of Verbascum (Abad et al., 2015). Our data confirm the presence of this species on Verbascum sp. The host plant of Tychius breviusculus are Medicago sp.; Melilotus sp.; Vicia sp. (Abad et al., 2015). However, it might feed on Alhagi sp., too. Tychius urbanus is generally collected on Alhagi maurorum, Alhagi camelorum (Abad et al., 2015). We collected this species for the first time on Alhagi officinalis. About 91 species of the subfamily Curculioninae have been recognized in Iran (Löbl and Smetana, 2013) while 199 species of this subfamily have been recorded to date from nearby Turkey (Löbl and Smetana, 2013). Therefore it can be anticipated that much more species will be found in Iran, necessitating further studies on the fauna of this subfamily.

\section{Acknowledgement}

The authors wish to thank Dr. Roberto Caldara for confirming the identification of collected species. 


\section{Literature}

Abad, R. G. G., Sadeghi, S. E., Ghajarieh, H., Nasserzadeh, H., Yarmand, H., Moniri, V. R. and Nikdel, M. (2015): Tychiini and Mecinini (Coleoptera : Curculionidae, Curculioninae) of Iran: eleven species recorded for the first time, with new data on host plants and distribution of several species. J. Ent. Soc. Iran, 35, 57-68.

Anderson, R. S. (2002): Curculionidae Latreille 1802. In: R. H. Arnett Jr., M. C. Thomas, P. E. Skelley and J. H. Frank (eds): American Beetles. Vol. 2, Polyphaga: Scarabaeoidea through Curculionoidea. CRC Press, Boca Raton, xiv+1-816 pages

Borumand, H. (1998): Insects of Iran. The list of Coleoptera in the insect collection of Plant Pests and Diseases Research Institute. Ministry of Agriculture, Agricultural Research, Education and Extension Organisation, Insect Taxonomy Research Department, Tehran, $116 \mathrm{p}$.

Caldara, R. (1985): Revisione delle Sibinia paleartiche (Coleoptera Curculionidae). Mem. Soc. Entomol. Ital., 63, 24-105.

Caldara, R. (1990): Revisione tassonomica delle specie paleartiche del genere Tychius Germar (Coleoptera Curculionidae). Mem. Soc. ital. Sci. nat. Mus. civ. Stor. nat. Milano, 25, 51-218.

Caldara, R. (2001): Phylogenetic analysis and higher classification of the tribe Mecinini. Koleopterol. Rundsch., 71, 171-203.

Caldara, R. (2014): Rhinusa Stephens: a taxonomic revision of the species belonging to the R.tetra and R. bipustulata groups (Coleoptera Curculionidae). J. Insect Biodiv. 2, 1-46.

Caldara, R. and Fogato, V. (2013): Systematics of the weevil genus Mecinus Germar, 1821 (Coleoptera : Curculionidae). I. Taxonomic treatment of the species. Zootaxa 3654, 1-105.

Caldara, R., Franz, N. and Oberprieler, R. G. (2014): 3.7. 10. Curculioninae Latreille, 1802. In: R. A. B. Leschen and R. G. Beutel (eds): Handbook of Zoology, Arthropoda: Insecta: Coleoptera. Vol. 3, Morphology and Systematics (Phytophaga). Walter de Gruyter, Berlin, pp. 589-628.

Dieckmann L. (1988): Beiträge zur Insektenfauna der DDR: Curculioninae. Beiträge zur Entomologie 38, $365-468$.

Egorov, A. B., Zherichin, V. V. and Korotyaev, B. A. (1996): Fam. Curculionidae - dolgonosiki ili sloniki [A key to Insects of the Russian Far East]. Vladivostok, Vol. 3. Pt. 3. pp. 249-311, 431-516. [In Russian].

Hoffmann, A. (1954): Coléoptères Curculionides (Deuxième partie). Faune de France, 29. Fédération Française des Sociétés de Sciences Naturelles, Paris, 1208 p.

Isaev, A. Yu. (1994): Ecologo-faunistic review of weevils (Coleoptera: Apionidae, Rhynchophoridae, Curculionidae) from Ul'janovsk Oblast'. Nature of Ul'janovsk Oblast', Ul'janovsk, 102 p.

Legalov, A. A., Ghahari, H. and Arzanov, Y. G. (2010): Annotated catalogue of curculionid-beetles (Coleoptera: Anthribidae, Rhynchitidae, Attelabidae, Brentidae, Brachyceridae, Dryophthoridae and Curculionidae) of Iran. Amurian Zoological J. 2, 191-244.

Löbl, I. and Smetana, A. (eds) (2013): Catalogue of Palaearctic Coleoptera. Vol. 8, Curculionidae. Brill, Leiden, $924 \mathrm{p}$.

Marvaldi, A. E. and Lanteri, A. A. (2005): Key to higher taxa of South American weevils based on adult characters (Coleoptera, Curculionoidea). Rev. Chil. Hist. Nat. 78, 65-87.

Modarres Awal, M. (2010): List of Agricultural Pests and their Natural Enemies in Iran. Ferdowsi University Press, Mashhad, $429 \mathrm{p}$.

Oberprieler, R. G., Marvaldi, A. E. and Anderson, R. S. (2007): Weevils, weevils, weevils everywhere. Zootaxa $1668,491-520$.

Smreczyński, S. (1976): Podrodzina Curculioninae. Plemiona: Nanophyini, Mecinini, Cionini, Anoplini, Rhynchaenini i uzupełnienia do zeszytów 98a-e. Klucze do oznaczania owadów Polski, cz. XIX. z. $98 \mathrm{f}$. Polskie Towarzystwo entomologiczne, Warszawa, 115 p. 